\title{
Eficacia y seguimiento en el largo plazo de pacientes con psoriasis vulgar moderada a severa en tratamiento con infliximab (Remicade ${ }^{\circledR}$ )
}

\author{
María del Pilar Valdés A, Francisca Schroeder H, \\ Vicky Roizen G, Juan Honeyman M, Leonardo Sánchez M. \\ Efficacy of infliximab in patients \\ with moderate and severe psoriasis \\ treated with infliximab (Remicade ${ }^{\circledR}$ )
}

Background: Psoriasis has a moderate or severe course in $25 \%$ of patients, requiring systemic therapy that is not always successful. Infliximab is a human-murine monoclonal anti tumor necrosis alpha (TNF- $\alpha$ ) antibody. This mediator has a role in the pathogenesis of psoriasis. Aim: To evaluate the use of infliximab in psoriatic patients resistant to conventional therapies. Material and Methods: An open prospective study including eight patients with extensive plaque or erythrodermic psoriasis. They were treated with infliximab $5 \mathrm{mg} / \mathrm{kg} / \mathrm{dose}$ on weeks 0,2 and 6 . Patients were evaluated every 2 weeks for a median lapse of 50.1 weeks. Physical examination, PASI scores (Psoriasis Area Severity Index) and a photographic control, were done in each visit. Results: All the patients responded in the first 10 weeks of follow-up. The mean reduction in PASI score was $86.6 \%$. Six patients received fourth infusion of infliximab at 37.3 weeks, on average. The most common adverse events were pruritus and headache. Conclusions: This group of patients with extensive plaque or erythrodermic psoriasis resistant to conventional therapies, had a good response to infliximab (Rev Méd Chile 2006; 134: 326-31).

(Key words: Antibodies, monoclonal; infliximab; psoriasis)

Recibido el 16 de julio, 2004. Aceptado el 13 de septiembre, 2005.

Departamento de Dermatología, Hospital Clínico, Universidad de Chile.

L

a psoriasis es una de las dermatosis más Lrecuentes en la práctica diaria, estimándose que afecta entre $2 \%$ y $3 \%$ de la población ${ }^{1}$.

Aproximadamente $25 \%^{2}$ de los pacientes psoriáticos presentan formas moderadas a severas,

Correspondencia a: Dra. Francisca Schroeder Hanke. Fono: (2) 2324489. E mail: lulateno@hotmail.com que afectan significativamente su calidad de vida, requiriendo en algún momento de su evolución tratamientos sistémicos para controlar su enfermedad. En éstos se utiliza ciclosporina, metotrexato, acitretin, radiación ultravioleta B (UVB) o radiación ultravioleta A asociado a psoralenos (PUVA).

La ciclosporina es un tratamiento muy efectivo, pero presenta riesgo conocido de hipertensión 
arterial y nefrotoxicidad que puede ser irreversible. El metotrexato demora 4 a 6 semanas para producir respuesta clínica y presenta riesgo de hepatotoxicidad y mielosupresión. Acitretin, como monoterapia en general, no logra una respuesta total en pacientes con psoriasis en placa extensa, los principales efectos adversos son mucocutáneos y es un medicamento reconocidamente teratogénico, que obliga a anticoncepción estricta hasta 2 a 3 años posterior a su suspensión. La fototerapia es un tratamiento efectivo, pero requiere 2 a 3 sesiones semanales por varios meses y requiere terapia de mantención.

La psoriasis es una enfermedad genéticamente definida, que se caracteriza por la hiperproliferación y diferenciación anormal de los queratinocitos, acompañada de un aumento de los marcadores de inflamación ${ }^{3}$.

La actividad inflamatoria en las placas psoriáticas se inicia, en parte, por la activación de linfocitos $\mathrm{T}$ en epidermis y dermis. Los linfocitos Th1 son los que predominan en las lesiones y liberan citoquinas proinflamatorias como el factor de necrosis tumoral $\alpha$ (TNF- $\alpha$ ), interleuquinas (IL) 6 y 8, interferón $\gamma$ y el factor estimulador de colonia granulocito-macrófago (GM-CSF).

El TNF- $\alpha$, a su vez, estimula la síntesis de numorosas citoquinas proinflamatorias, incluyendo IL 6 y 8 y GM-CSF; favorece la activación de los linfocitos T por parte de las células de Langerhans; induce la síntesis de moléculas de adhesión (ICAM1, VCAM-1) en las células endoteliales y en queratinocitos, favoreciendo la llegada de células inflamatorias a la piel; promueve la proliferación de queratinocitos y estimula al queratinocito psoriático a producir el factor de crecimiento vascular endotelial (VEGF) promoviendo la angiogénesis ${ }^{4}$.

Todo lo señalado anteriormente apoya el rol fundamental que tiene el TNF- $\alpha$ en la patogenia de la psoriasis y ha llevado a plantear que bloqueando su acción disminuiría la proliferación de queratinocitos y la inflamación, ambos pilares en el desarrollo de las lesiones psoriáticas.

Infliximab (Remicade ${ }^{\circledR}$ ) es un anticuerpo monoclonal de tipo IgG1 quimérico (humano/murino) anti TNF- $\alpha$. Infliximab bloquea la acción del TNF- $\alpha$ uniéndose a su forma soluble o unida a membranas celulares. El TNF- $\alpha$ unido a las moléculas de infliximab no puede unirse ni activar al receptor de TNF- $\alpha^{2-4}$.
Se ha utilizado exitosamente en el manejo de enfermedades mediadas por TNF- $\alpha$, como enfermedad de Crohn y artritis reumatoide. En 1998, la Food and Drug Administration de los Estados Unidos de Norteamérica (FDA) aprobó su uso en enfermedad de Crohn moderada a severa rebelde a terapias habituales y en 1999 se aprobó su uso en forma simultánea con metotrexato en artritis reumatoide, en pacientes que no respondían a monoterapia.

Existen múltiples reportes ${ }^{2,4-7}$ de su efectividad en pacientes psoriáticos, siendo el objetivo de nuestro trabajo evaluar su efectividad en pacientes chilenos con psoriasis extensa resistente a terapias convencionales.

\section{MATERIAL Y MÉTODO}

Se realizó un estudio prospectivo, abierto, siguiendo a 8 pacientes adultos con historia de psoriasis en placa extensa o enitrodérmica tratados con infliximab endovenoso. Se definió como psoriasis en placa extensa aquella que comprometía más de $20 \%$ de superficie corporal y como psoniasis enitrodérmica aquella que comprometía más de $75 \%$ de la superficie corporal. En ambos casos, el cuadro cutáneo afectaba significativamente la calidad de vida de los pacientes.

Este estudio se llevó a cabo en el Servicio de Dermatología del Hospital Clínico de la Universidad de Chile, contando con la aprobación del Comité de Etica de dicho hospital.

Los criterios de inclusión fueron: pacientes adultos con historia de psoriasis de al menos 6 meses de evolución, sin respuesta a tratamientos de primera y segunda línea (metotrexato, retinoides orales y fototerapia), o que han presentado reacciones adversas que han obligado a la suspensión de éstos.

Los criterios de exclusión fueron: niños y adolescentes; mujeres embarazadas o en lactancia; antecedentes de hepatitis, infección respiratoria baja o pielonefritis aguda en los últimos 3 meses; tuberculosis activa; pacientes portadores del virus VIH; pacientes inmunosuprimidos o en etapa de SIDA; pacientes con serología positiva para VHB y VHC; pacientes portadores de neoplasia maligna; alcoholismo o drogadicción.

Los pacientes fueron informados detalladamente del estudio y firmaron un consentimiento 
informado, en el que se les explicó los mecanismos de acción y posibles reacciones adversas del medicamento a utilizar.

Previo al inicio del tratamiento se realizó historia clínica y examen físico consignando el índice de PASI (Psoriasis Area and Severity Index). Este índice es un parámetro de medición del grado de actividad de la psoriasis que evalúa tres parámetros; eritema, infiltración y descamación, en las 4 áreas corporales; cabeza, tronco, extremidades superiores y extremidades inferiores ${ }^{8}$. El PASI tiene un máximo de 72 puntos y un mínimo de 0 punto. Además se les tomó fotografías y se les solicitaron los siguientes exámenes: hemograma y VHS; perfil bioquímico completo; perfil hepático; orina completa; anticuerpos antinucleares (ANA); anticuerpos antiADN nativo; VIH y serología VHB y VHC; radiografía de tórax.

Previo al inicio del tratamiento se les suspendió cualquier tratamiento tópico o sistémico que hubiesen estado recibiendo para manejar su enfermedad.

Se administró infliximab en dosis de $5 \mathrm{mg} /$ $\mathrm{kg} /$ dosis en las semanas 0,2 y 6 . El medicamento se administró en forma endovenosa, diluido en 250 cc de solución salina isotónica a pasar en $2 \mathrm{~h}$ con el paciente hospitalizado, bajo supervisión continua. Los pacientes fueron dados de alta $2 \mathrm{~h}$ posterior al término de la infusión endovenosa.

Los pacientes fueron controlados cada dos semanas, por un peníodo mínimo de 6 meses, en el policlínico de psoriasis del Servicio de Dermatología del Hospital Clínico de la Universidad de Chile. En cada control se realizó anamnesis y examen físico completo con medición del PASI y se interrogó dirigidamente buscando posibles reacciones adversas. Se tomaron fotografías.

Al cumplirse 10 semanas de tratamiento se solicitaron exámenes de control (hemograma, VHS, perfil bioquímico y hepático, orina completa, ANA).

Los pacientes que presentaron recaída de su cuadro psoriático alcanzando $50 \%$ del PASI basal, y que habían respondido al tratamiento, recibieron una dosis de refuerzo de $5 \mathrm{mg} / \mathrm{kg} /$ dosis iv.

\section{Resultados}

Se incluyó una población de 8 adultos portadores de psoriasis ( 5 varones y 3 damas). Tres pacientes con psoriasis eritrodérmica (pacientes №1, №3 y №5) y cinco con psoriasis en placa extensa (pacientes №2, №4，№6，№7， №8). La edad promedio fue de 46 años, con rango de 23 a 60 años. Un paciente (№2) abandonó el seguimiento a las 9 semanas de iniciada la terapia por estar insatisfecho con el resultado del tratamiento.

El PASI basal promedio fue de 30,8 con un rango de 16,4 a 42,6. La evolución del PASI durante las primeras 10 semanas de seguimiento se observa en la Tabla 1.

Se definió como paciente respondedor al tratamiento aquel que presentó un descenso del PASI basal de al menos 75\%.

El total de los pacientes (8 de 8) fueron respondedores al tratamiento dentro de las primeras 10 semanas de iniciada la terapia. En la semana 10 observamos una disminución del PASI promedio de 30,8 a 3,5, es decir, de 88,6\%.

El tiempo promedio de respuesta al tratamiento fue de 5,7 semanas, es decir, el tiempo promedio en que los pacientes alcanzaron un descenso del PASI $\geq 75 \%$ del PASI basal.

La evolución en el PASI promedio a lo largo de las primeras 16 semanas de seguimiento se observa en la Figura 1.

El objetivo principal de nuestro estudio fue el seguimiento en el largo plazo, lo que se definió como mayor a seis meses y se pudo realizar en 7 de los 8 pacientes. El tiempo promedio de seguimiento fue de 50,1 semanas, con un rango entre 9 y 91 semanas.

Tabla 1. Evolución del PASI durante primeras 10 semanas de seguimiento en pacientes psoriáticos tratados con infliximab

\begin{tabular}{|ccccccc|}
\hline $\begin{array}{c}\text { Paciente } \\
\text { № }\end{array}$ & $\begin{array}{c}0 \\
\text { sem }\end{array}$ & $\begin{array}{c}2 \\
\text { sem }\end{array}$ & $\begin{array}{c}4 \\
\text { sem }\end{array}$ & $\begin{array}{c}6 \\
\text { sem }\end{array}$ & $\begin{array}{c}8 \\
\text { sem }\end{array}$ & $\begin{array}{c}10 \\
\text { sem }\end{array}$ \\
\hline 1 & 40,8 & 6,3 & 1,7 & 3,9 & 2,4 & 5,6 \\
2 & 32,1 & 2,2 & 4,2 & 30,5 & 12,5 & \\
3 & 42,6 & 13,7 & 5,2 & 2,8 & 2,8 & 1,3 \\
4 & 16,4 & 16,4 & 10 & 6,2 & 3,6 & 3 \\
5 & 40,1 & 14,8 & 17,8 & 12,3 & 9,7 & 10,2 \\
6 & 33,1 & 17,1 & 15,2 & 15,2 & 10 & 3,6 \\
7 & 22,5 & 13,4 & 8,3 & 6,5 & 3,1 & 0,8 \\
8 & 19,2 & 10,6 & 2,4 & 0,8 & 0 & 0 \\
$\bar{x}$ & 30,8 & 11,8 & 8,1 & 9,7 & 5,5 & 3,5 \\
\hline
\end{tabular}




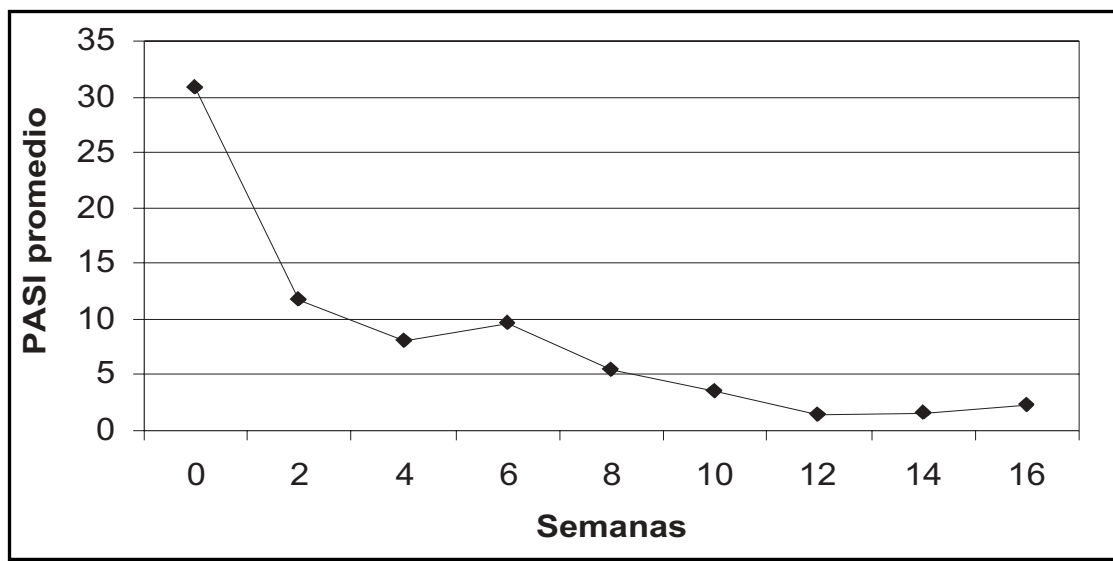

FIGURA 1. Evolución del PASI promedio durante primeras 16 semanas de seguimiento.

A las 4 semanas de iniciado el tratamiento, se observa un descenso del PASI promedio desde 30,8 (PASI basal) a 8,1.

De los 6 pacientes que requirieron dosis de refuerzo, se puede observar que el tiempo necesario para alcanzar $50 \%$ del PASI basal (momento definido para la aplicación de la dosis de refuerzo), fue en promedio 37,3 semanas, con rango entre 22 y 50 semanas.

Es importante destacar que durante el seguimiento los pacientes no recibieron ningún otro tratamiento sistémico para el manejo de la psoriasis. En relación a tratamientos tópicos, sólo se indicaron cremas humectantes hasta la semana 20. A partir de la semana 20 se indicaron corticoides tópicos, en algunos pacientes, con el fin de lograr retrasar el momento necesario para la aplicación de la dosis de refuerzo.

La respuesta a las dosis de refuerzo se clasificó en: Buena: descenso del PASI $\geq 75 \%$; Regular: descenso del PASI entre 50\% y 75\%; Mala: descen- so del PASI entre $25 \%$ y $50 \%$; Sin respuesta: descenso del PASI $<25 \%$.

La respuesta a las dosis de refuerzo, se detalla en la Tabla 2.

El paciente de mayor seguimiento (paciente №1) lleva 91 semanas y ha requerido 4 dosis de refuerzo (sem 26, 41, 61 y 84), presentando buena respuesta. Su evolución se observa en la Figura 2.

Los eventos adversos observados en nuestros pacientes se resumen en la Tabla 3.

La paciente №3 debió abandonar el estudio a las 39 semanas de seguimiento por presentar un carcinoma ductal de mama. La paciente tiene mamografía y ecotomografía mamaria normal cinco meses previos al diagnóstico del cáncer de mama (18 semanas de seguimiento). Se realizó tumorectomía y vaciamiento axilar (ganglios negativos) y está

Tabla 2. Respuesta a dosis de esfuerzo

\begin{tabular}{|cccccc|}
\hline $\begin{array}{c}\text { Paciente } \\
\text { No }\end{array}$ & $\begin{array}{c}\text { № dosis } \\
\text { de refuerzo }\end{array}$ & $\begin{array}{c}\text { Respuesta } \\
\text { buena }\end{array}$ & $\begin{array}{c}\text { Repuesta } \\
\text { regular }\end{array}$ & $\begin{array}{c}\text { Respuesta } \\
\text { mala }\end{array}$ & $\begin{array}{c}\text { Sin } \\
\text { respuesta }\end{array}$ \\
\hline 1 & 4 & 3 & 1 & - & - \\
4 & 2 & - & - & - & 2 \\
5 & 3 & 1 & 2 & - & - \\
6 & 1 & - & 1 & - & - \\
7 & 1 & - & - & 1 & - \\
8 & 1 & - & - & & \\
\hline
\end{tabular}




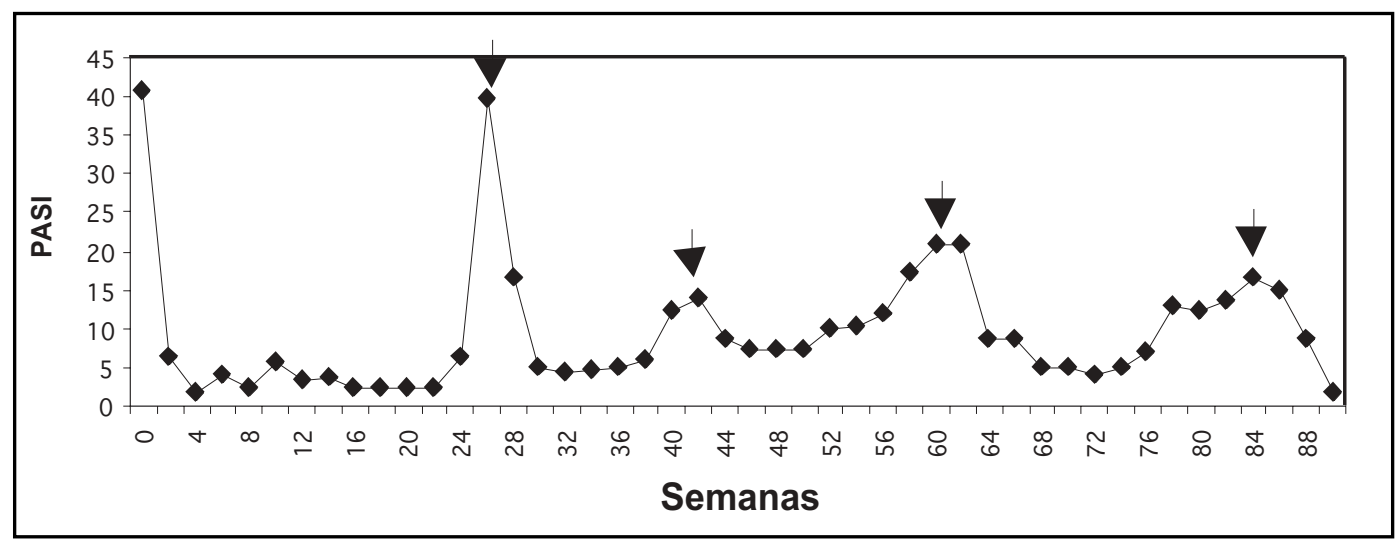

FIguRA 2. Evolución del PASI paciente №1.

Tabla 3. Eventos adversos

\begin{tabular}{|lc|}
\hline Evento adverso & № de pacientes \\
\hline Fatiga & 3 \\
Cefalea & 2 \\
Artralgias & 2 \\
Alopecia difusa & 2 \\
Prurito & 3 \\
Mialgias & 1 \\
Infecciones respiratorias & 1 \\
Síndrome lupus like & 0 \\
Neoplasias & 1 \\
Anafilaxia & 0 \\
\hline
\end{tabular}

en tratamiento con radioterapia y quimioterapia. Este evento adverso fue reportado al laboratorio.

Se presentó alopecia difusa en 2 pacientes, entre las semanas 14 y 16 de seguimiento. Ambos pacientes presentaron tricograma normal y resolución espontánea de la alopecia.

Un paciente presentó infección respiratoria baja, neumonitis, a la semana 12 de seguimiento, tratada en forma ambulatoria con antibióticos orales, con buena respuesta al tratamiento y resolución de cuadro respiratorio sin descompensación cutánea.

No observamos en nuestros pacientes reacciones agudas significativas relacionadas con la infusión iv, 2 pacientes presentaron cefalea y 3 pacientes presentaron fatiga en las 24 a 48 horas posterior a la infusión.

No se observaron alteraciones de los exámenes de laboratorio (hemograma, VHS, perfil bio- químico, perfil hepático, orina completa, ANA) las 10 semanas de seguimiento.

\section{DisCUSIÓN}

Nuestros resultados demuestran que infliximab es efectivo en el tratamiento de la psoriasis. El total de los pacientes (8/8) fueron respondedores al tratamiento dentro de las primeras 10 semanas de iniciada la terapia. Definiendo como paciente respondedor aquel que presentara un descenso del PASI $\geq 75 \%$ del PASI basal.

En la semana 10 observamos una disminución del PASI promedio de 30,8 a 3,5, es decir, de 88,6\%.

$\mathrm{Al}$ comparar nuestros resultados con las publicaciones anteriores respecto a infliximab y psoriasis, observamos que los resultados son similares. Chaudhari, Gottlieb y cols ${ }^{2}$, observaron que $81,8 \%$ de los pacientes (9 de 11) del grupo tratado con infliximab $5 \mathrm{mg} / \mathrm{kg} /$ dosis iv (semana 0, 2 y 6) alcanzaron un descenso del PASI $\geq 75 \%$ del PASI basal. En la semana 10 observaron una disminución del PASI promedio de 22,1 a 3,8, es decir, de 82,8\%.

En otro trabajo similar, Schopf y $\operatorname{cols}^{6}$, que trataron a 8 pacientes con psoriasis moderada a severa, con infliximab $5 \mathrm{mg} / \mathrm{kg} /$ dosis iv en la semana 0,2 y 6 . Observaron que $87,5 \%$ de los pacientes $/ 7$ de 8) presentaron un descenso del PASI $\geq 75 \%$ del PASI basal a la semana 10 . En la semana 10 observaron una disminución del PASI promedio de 21,75 a 3,4 , es decir, de $84,3 \%$.

El objetivo principal de nuestro estudio fue el seguimiento en el largo plazo, lo que se definió 
como mayor a seis meses y se pudo realizar en el $87,5 \%$ ( 7 de 8 pacientes) de los pacientes. Durante el seguimiento, los pacientes fueron controlados cada 2 semanas con el índice PASI y se les indicó dosis de refuerzo de $5 \mathrm{mg} / \mathrm{kg} /$ dosis iv a aquellos pacientes que presentaron una recaída de su cuadro psoriático alcanzando 50\% del PASI basal. Observamos que el tiempo promedio para alcanzar 50\% del PASI basal, momento definido para recibir la dosis de refuerzo, fue de 37,3 semanas. Seis pacientes recibieron dosis de refuerzo. Tres de ellos presentaron una respuesta buena a regular a la dosis de refuerzo y 3 presentaron una respuesta mala a nula.

Es importante destacar que durante el seguimiento los pacientes no recibieron ningún otro tratamiento sistémico para el manejo de la psoriasis. Sin embargo, a partir de la semana 20 se indicaron corticoides tópicos en algunos pacientes, con el fin de lograr retrasar el momento necesario para la aplicación de la dosis de refuerzo, dado el alto costo de este medicamento.

En relación a los 3 pacientes no respondedores a la dosis de refuerzo, cabe plantearse la posibilidad de refractariedad al medicamento por producción de anticuerpos anti-infliximab (los cuales no se pudieron medir, ya que no está disponible este examen en Chile), como también la posibilidad de que una dosis sea insuficiente para controlar la recaída de la enfermedad. Sería

\section{REFERENCIAS}

1. SÁnchez L Aspectos Epidemiológicos en Psoriasis. En: Sánchez L. Psoriasis. 1a Edición, Santiago: Actualidades Médicas, 1999; 13-20.

2. Chaudhari U, Romano P, Mulcahy LD, Dooley LT, BAKER DG, GotTLEB AB. Efficacy and safety of infliximab monotherapy for plaque-type psoriasis: a randomized trial. Lancet 2001; 357: 1842-7.

3. Honeyman J. Aspectos Fisiopatológicos de la Psoriasis. En: Sánchez L. Psoriasis. 1a Edición, Santiago: Actualidades Médicas, 1999; 21-96.

4. Gоттleв A. Infliximab for psoriasis. J Am Acad Dermatol 2003; 49: S112-7.

5. Gottleb AB, Chaudhari U, Mulcahy LD, L S, Dooley interesante administrar como refuerzo el ciclo inicial de tratamiento (3 dosis).

$\mathrm{Al}$ analizar la evolución en el largo plazo de los pacientes respondedores a las dosis de refuerzo, se puede observar que requieren dosis de refuerzo aproximadamente cada 20 semanas, lo que implica que infliximab podría ser un tratamiento de mantención, aplicándose 2 a 3 veces al año según necesidad. Este hallazgo coincide con lo observado en pacientes con artritis reumatoide, en los que está comprobado su efectividad como tratamiento a largo plazo con dosis de mantención cada 8 semanas. Hay que destacar que en los pacientes con artritis reumatoide, infliximab se indica asociado a metotrexato. En el futuro sería interesante comparar la respuesta al tratamiento con monoterapia de infliximab versus infliximab más metotrexato en psoriasis ${ }^{7}$.

Los eventos adversos presentados fueron en general leves, salvo una paciente que presentó un carcinoma ductal de mama. No queda claro la relación entre este fármaco y la enfermedad, situación que debe aclararse en estudios posteriores.

En resumen, infliximab constituye una buena alternativa terapéutica para pacientes con psoriasis extensa o eritrodérmica, refractarias a tratamiento de primera y segunda línea. Su seguridad a largo plazo debe ser confirmada en estudios con mayor número de pacientes y mayor seguimiento.

LT, BAKER DG. Infliximab monotherapy provides rapid and sustained benefit for plaque-type psoriasis. J Am Acad Dermatol 2003; 48: 829-35.

6. Schopf R, Aust H, KNop J. Treatment of psoriasis with the chimeric monoclonal antibody against tumor necrosis factor $\alpha$, infliximab. J Am Acad Dermatol 2002; 46: 886-91.

7. Kirby B, Marsland AM, Carmichael AJ, Griffiths CEM. Successful treatment of severe recalcitrant psoriasis with combination infliximab and methotrexate. Clin Exp Dermatol 2001; 26: 27-9.

8. Fredriksson T, Pettersson U. Severe Psoriasis Oral Therapy with a New Retinoid. Dermatologica 1978; 157: 238-44.

El laboratorio Schering-Plough aportó la mitad de los medicamentos administrados a los pacientes. 